\title{
THE ROLE OF THE SOURCE OF SOCIAL INFORMATION IN THE FORMATION OF PLACEBO ANALGESIA
}

Authors: Elżbieta A. Bajcar, Karolina Wiercioch-Kuzianik, Dominika Farley, Ewa Buglewicz, Helena Bieniek, Justyna Brączyk, Przemysław Bąbel

Jagiellonian University, Institute of Psychology, Pain Research Group, Kraków, Poland

Wagtey IN KRAKOWW

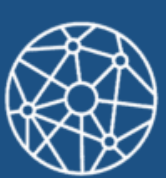

INSTITUTE OF PSYCHOLOGY JAGIELLONIAN UNIVERSITY
TAIN

RTSEARCH

GRTUP
NATIONAL SCIENCE CENTRE

\section{AIMS OF INVESTIGATION}

Previous studies showed that observing pain behavior displayed by other people may modify pain experiences of the observer. Moreover, changes in pain experiences may be induced even when the individual has neither direct (observation in vivo) nor indirect (video recording) contact with other people experiencing pain but familiarizes him or herself with pain ratings provided by those people instead. This study aimed to examine whether:

$\checkmark$ vicariously provided information about pain is able to elicit placebo analgesia

$\checkmark$ information coming from a group of people is more effective in shaping placebo analgesia than information coming from a single individual,

$\checkmark$ information coming from individuals previously observed in the same experimental situation produces a stronger placebo effect than information coming from anonymous participants.

\section{Participants}

\section{METHODS}

$\checkmark 118$ healthy volunteers $(60 \%$ females), age range $18-32$ years (mean $=22,4 \pm 3,15)$

$\checkmark$ Exclusion criteria: (1) age below 18 and over 35, (2) previous participation in a pain study, (3) pain complaints, (4) taking painkillers, (5) using drugs, (6) overusing alcohol, (7) presence or history of any neurological, respiratory, circulatory, musculoskeletal and/or psychiatric disorders.

\section{Stimuli}

$\checkmark$ Pain stimulus: thermal heat pain delivered to the volar surface of the nondominant forearm. Apparatus: Pathway Pain \& Sensory Evaluation Sysytem (Medoc, model ATS). The intensity of thermal stimuli was calculated individually for each participant based on the calibration procedure. Pain threshold and pain tolerance were determined four times. Last three out of four obtained values were averaged for $t$ and $T$, respectively. Pain intensity of the stimuli was set at: $t+(T-t)^{*} 0.75$

$\checkmark$ Visual stimulus: white circle presented in full-screen mode on a computer screen (17", resolution $1280 \times 1024)$ facing the participant at a distance of approximately $50 \mathrm{~cm}$

\section{Measures}

$\checkmark$ Pain intensity measured on a visual analogue scale (VAS) ranged from 'no pain' to 'the most intensive pain that is tolerable'.

\section{Design and procedures}

Randomization: Participants were randomly assigned to one of four experimental groups and one control group. Participants from experimental groups 1 and 3 were presented with pain ratings which supposedly came from an individual (another participant), while participants from experimental groups 2 and 4 were presented with pain ratings supposedly provided by a group of eight people (other participants). Participants from groups 3 and 4 had also previously watched a video recording showing individuals who ostensibly provided those pain ratings. Participants in the control group did not receive any pain-related information.

Pre-test: Participants received a series of 8 thermal pain stimuli of the same temperature, individually set for each participant. Participants rated the intensity of pain. No additional visual stimuli were presented at this stage.

Manipulation phase: Participants from the experimental groups received information on how other people who allegedly underwent the same experimental procedure rated the intensity of pain stimuli preceded by a white circle and pain stimuli applied without a visual cue. The pain ratings of the alleged participants were presented as vertical bars on the VAS, where each bar represented a rating of one participant. The ratings of pain stimuli presented with the visual stimulus (white circle) were always lower than those presented without it. The position of pain ratings was calculated individually for each participant to be proportional to his or her own ratings from the pre-test phase.

Post-test: Participants received a series of 16 thermal stimuli of the same temperature. Half of the stimuli were preceded by a white circle, and the other half were delivered without any visual cues. Participants rated the intensity of pain.

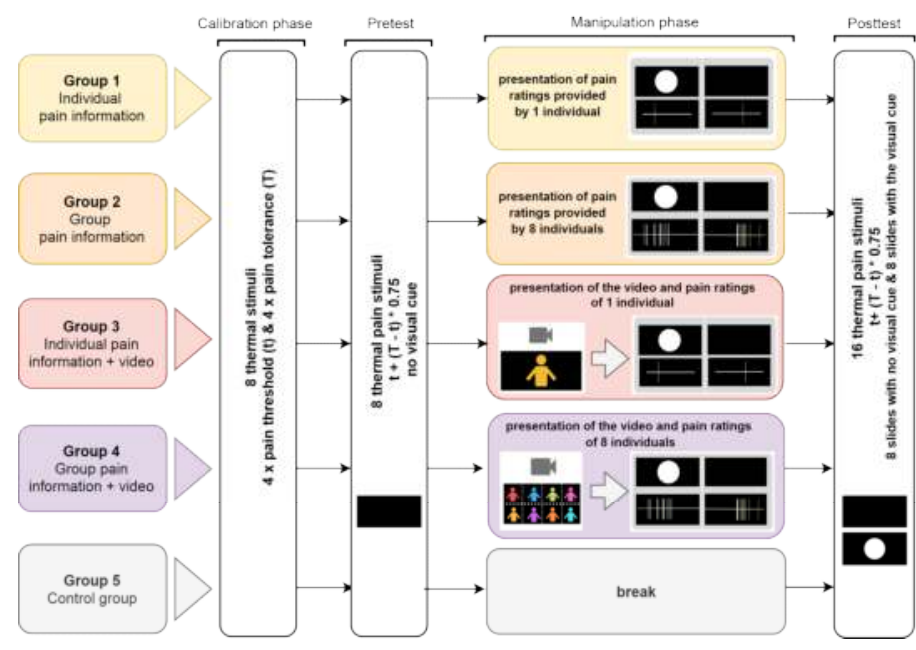

Fig. 1. Study design

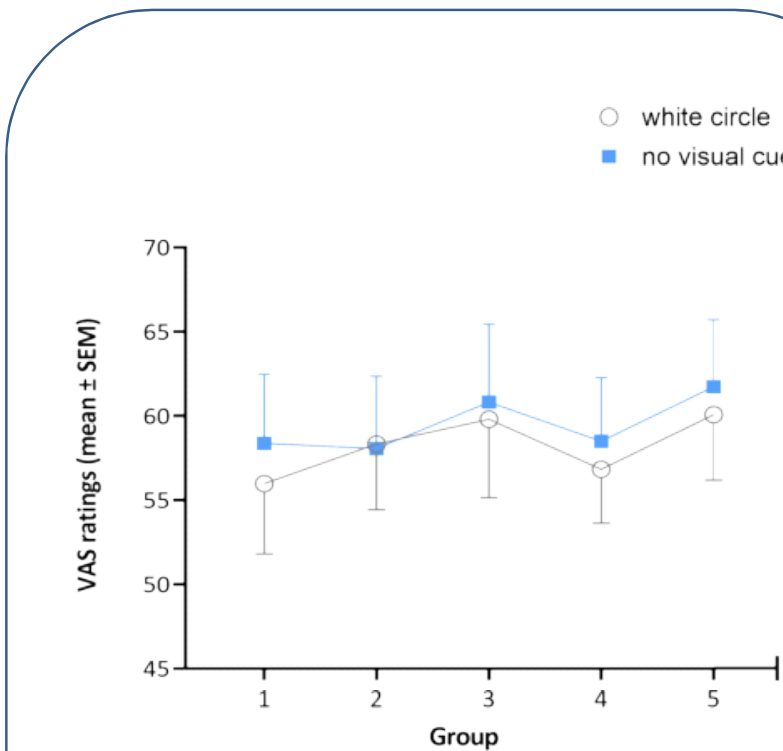

\section{RESULTS}

Participants in all experimental groups and the control group rated the intensity of experienced pain following presentation of the

white circle as lower than the intensity of pain experienced without any visual cues being shown.
There were no differences between experimental groups where ratings supposedly provided by an individual participant were There were no differences between experimental groups where ratings supposediticipants were shown.
shown versus the groups where ratings of a group of participant

There were no differences between experimental groups where video recordings of a model or models pretending to be participants were shown versus the groups where no video recordings were shown.

\section{CONCLUSIONS}

$\checkmark$ Information about pain intensity ratings provided by other people did not influence participants' pain ratings, regardless of whether that information was 1) provided by an individual or a group of people; 2) provided by anonymous or previously seen people. Presentation of a visual cue was associated with lower pain ratings, regardless of whether or not it was suggested to participants that it would be associated with lower pain stimulus intensity. Further studies are needed in order to elucidate why presentation of a visual cue alone would cause the pain ratings to drop. 